\title{
ANEURISMA CALCIFICADO DE LA ARTERIA RENAL EN PACIENTE MONORRENA, UNA PATOLOGÍA INUSUAL
}

\author{
J. RODRÍGUEZ CORCHERO, J. MARTÍN CALERO, J. MARTÍNEZ RODRÍGUEZ, \\ I. HUESA MARTÍNEZ, F. GARCÍA MATILLA
}

Servicio de Urología. Unidad de Litiasis. Hospital Universitario Virgen del Rocío. Sevilla.

Actas Urol Esp. 28 (9): 672-676, 2004

\section{RESUMEN}

ANEURISMA CALCIFICADO DE LA ARTERIA RENAL EN PACIENTE MONORRENA, UNA PATOLOGÍA INUSUAL

Los aneurismas de la arteria renal son infrecuentes. La prevalencia real de los mismos en la población general es desconocida, aunque se estima que es inferior al $0,4 \%$. Con el uso cada vez mayor de la Angiografia y la Tomografia Computerizada (TC), así como por la mejora en las técnicas de imagen, cada vez se diagnostican con más frecuencia. Las causas más comunes son la displasia fibromuscular y la oclusión arteriosclerótica de la arteria renal. Generalmente no hay una clínica patognomónica de los aneurismas renales, produciendo síntomas inespecíficos como dolor en el costado, hematuria, hipertensión e hipotensión (sospecha de rotura).

Presentamos el caso de una paciente monorrena con aneurisma calcificado en la arteria renal.

PALABRAS CLAVE: Aneurisma calcificado. Arteria renal. Monorreno.

\section{ABSTRACT \\ RENAL ARTERY CALCIFIED ANEURYSM IN A SOLYTARY KIDNEY WOMAN PATIENT, RARE PATHOLOGY}

Renal artery aneurysm are uncommon. The true prevalence of renal aneurysms in the general population is unknown (less than 0.4\%). Because of more widespread use of Angiography and CT as well as improved imaging techniques, they are diagnosed more frecuently. Fibromuscular dysplasia and arteriosclerotic occlusion of the renal artery are believed to be the most frecuent causes. In general, there are no pathognomonic signs and symptoms of renal aneurysm. Nonspecific complaints include flank pain, hematuria, hypertension and hypotension (suspect rupture of aneurysm).

We report a case of a woman with a renal artery calcified aneurysm in a solitary kidney.

KEY WORDS: Calcified aneurysm. Renal artery. Solitary kidney.

$\mathrm{L}$ os aneurismas de la arteria renal son dilataciones localizadas de la misma o de sus ramas causados por la debilidad del tejido elástico y de la capa media arterial. La prevalencia real de los mismos en la población general es desconocida, aunque se estima que es inferior al $0,4 \%$. Las causas más frecuentes son la displasia fibromuscular y la oclusión arteriosclerótica de la arteria renal. Con el uso cada vez mayor de la Angiografía y la Tomografía Axial Computerizada (TAC), así como por la mejora en las técnicas de imagen, cada vez se diagnostican con más frecuencia. Generalmente son asintomáticos aunque en ocasiones pueden producir síntomas inespecíficos como dolor en el costado, hematuria, hipertensión e incluso hipotensión brusca con shock hipovolémico en caso de rotura. 


\section{CASO CLÍNICO}

Paciente de 63 años estudiada en nuestras consultas por litiasis renal derecha. No presenta alergias conocidas ni enfermedades crónicas, destacando como único antecedente de interés el haber sido nefrectomizada hace 40 años (nefrectomía izquierda por tuberculosis).

\section{Historia actual}

Mujer revisada en nuestras consultas por presentar desde hace más de 20 años litiasis renal derecha con algunos episodios de cólicos nefríticos.

En 1998 se realiza un TAC en la que se objetiva una imagen de un centímetro de diámetro, adyacente a la pelvis renal derecha aunque sin contacto con el sistema excretor, que provoca una discreta hidronefrosis, sugestiva de divertículo pélvico.

En 1999 se hace una urografia intravenosa (UIV) informada como "imagen ovoidea en hilio renal con calcificaciones periféricas, de $22 \times 18$ $\mathrm{mm}$ sugestiva de aneurisma de la arteria renal derecha" (Fig. 1).

Posteriormente se realizan ecografias que se informan como litiasis pélvica que provoca ligera ectasia pielocalicial.

En septiembre del 2002 se hace un TAC (Fig. 2) que informa la imagen como sugestiva de aneurisma de la arteria renal, recomendando realizar una angio-TAC helicoidal con contraste que muestra la imagen antes mencionada, de $20 \times 17 \mathrm{~mm}$, sugestiva de aneurisma de la arteria renal derecha o una de sus ramas (Fig. 3).

Tras hacer una revisión exhaustiva de la literatura, le proponemos a la paciente realizarse una arteriografía para completar el estudio, negándose a dicha exploración. Ante esta situación, y al tratarse de una paciente monorrena, comentamos el caso con el servicio de Cirugía Vascular que no son partidarios de un tratamiento quirúrgico. Tras plantearle a la señora las ven-
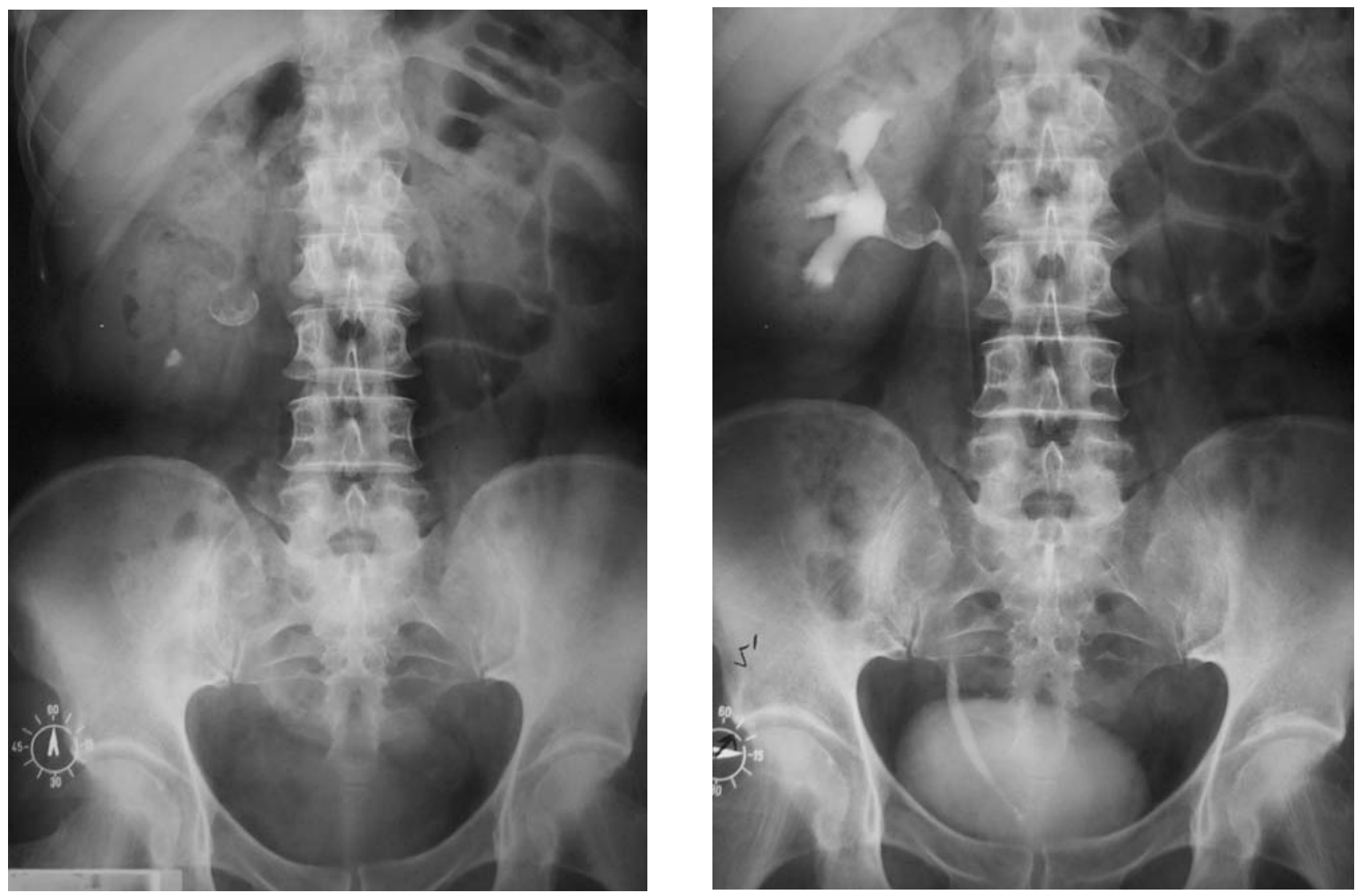

FIGURA 1. UIV: Imagen ovoidea en hilio renal derecho con calcificaciones periféricas, de $22 \times 18$ mm sugestivas de aneurisma de la arteria renal. 

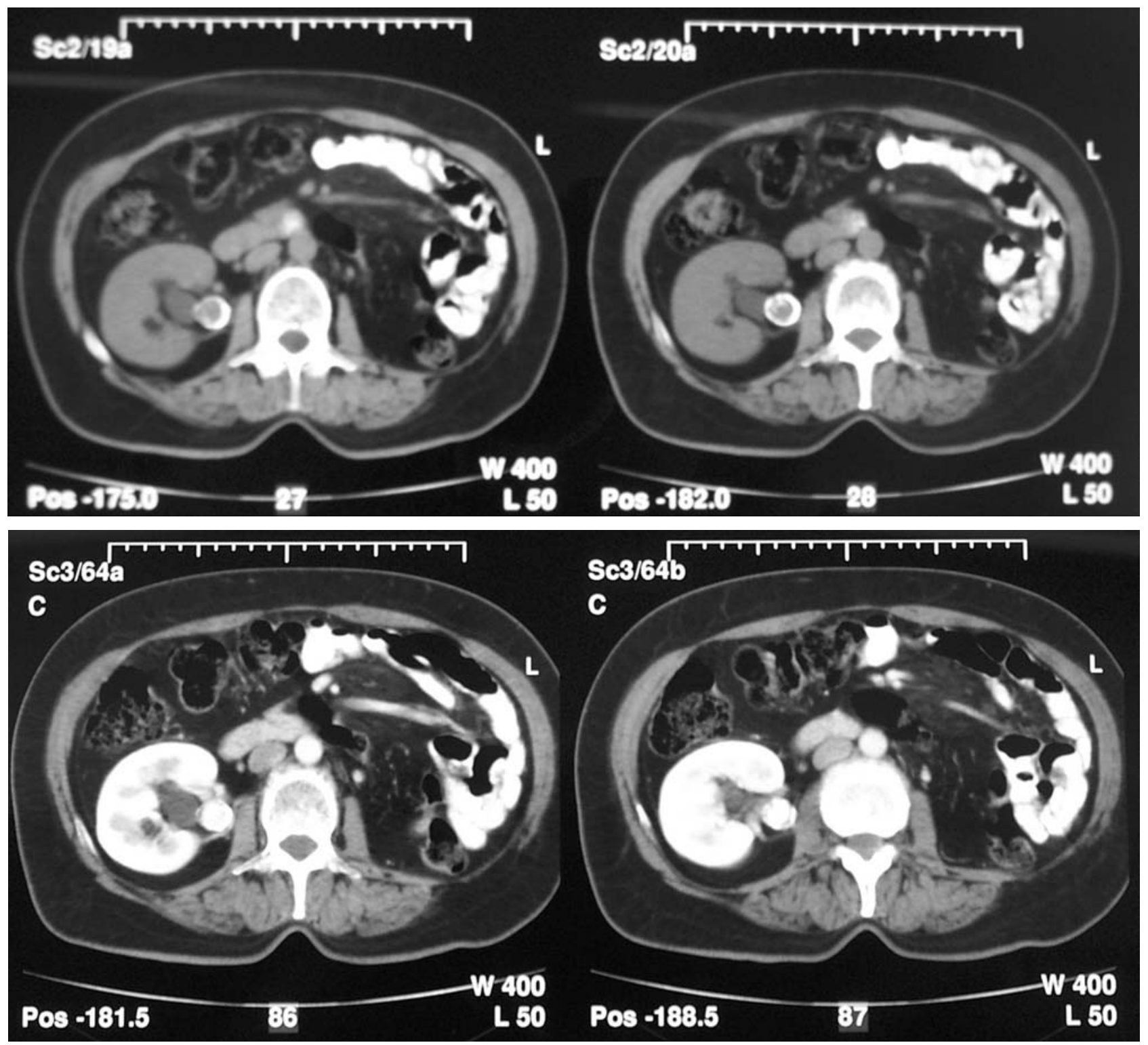

FIGURA 2. TAC sin y con contraste: Imagen sugestiva de aneurisma calcificado de la arteria renal en riñón único.

tajas e inconvenientes de las diferentes opciones terapéuticas, se decide actitud expectante.

\section{Evolución}

La paciente permanece asintomática, sin hipertensión arterial (HTA) ni dolor, siendo revisada periódicamente en nuestras consultas.

\section{COMENTARIOS}

Los aneurismas de la arteria renal son lesiones raras localizadas en la arteria renal principal o en una de sus ramas. Su incidencia es muy baja, constituyendo el $1 \%$ de todos los aneurismas ${ }^{1,2}$.
Según la clasificación de Poutasse hay cuatro tipos: sacular, fusiforme, disecante e intrarrenal, apareciendo con más frecuencia el primero (75\%), siendo de este tipo el que presentamos en el caso que nos ocupa.

Los aneurismas saculares renales se suelen localizar a nivel de la bifurcación de la arteria renal, donde la pared es más débil, asociándose en ocasiones a degeneración aterosclerótica y calcificación intramural. Cuando la calcificación es incompleta y la pared fina puede dar lugar a puntos de ulceración entre las placas calcificadas que en ocasiones se rompen de forma espontánea ${ }^{1}$. 

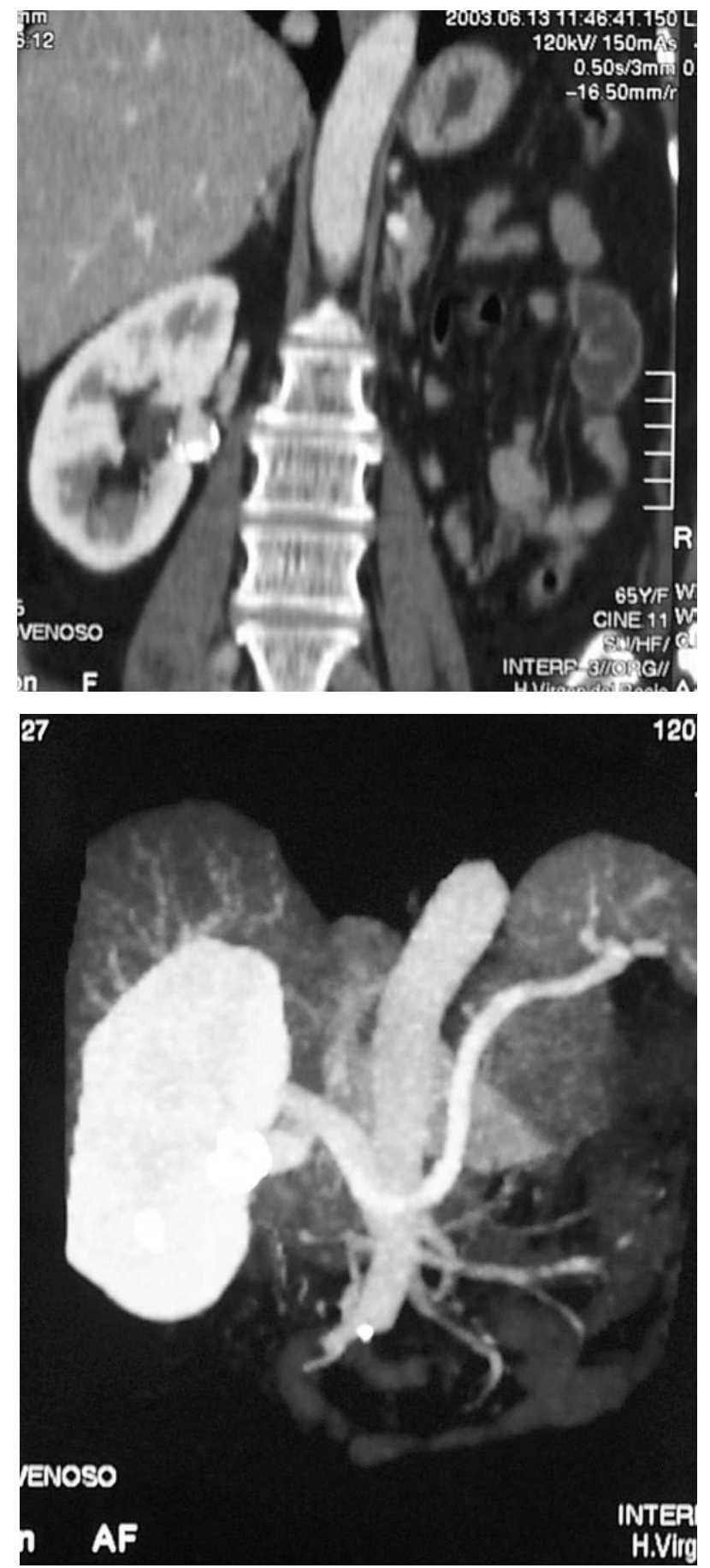

FIGURA 3. Angio TAC helicoidal con contraste: "imagen de $20 \times 17 \mathrm{~mm}$ con calcificaciones periféricas, sugestiva de Aneurisma de la Arteria renal derecha o una de sus ramas.

También está descrita la trombosis intra-aneurismática con embolización periférica.

La mayor parte de los aneurismas de la arteria renal suelen ser pequeños y asintomáticos ${ }^{2}$.
Las manifestaciones clínicas más frecuentes son: HTA, dolor subcostal o en flanco, hematuria y excepcionalmente una masa palpable. La hipertensión vasculorrenal aparece en un $20-70 \%$, siendo debida a estenosis asociada, disección, formación de fístula arteriovenosa, tromboembolismo o compresión de ramas vecinas por el propio aneurisma. La complicación más grave es la rotura $^{3,4}$ del aneurisma que puede dar lugar a hematuria macroscópica si se rompe hacia la vía urinaria o a dolor en flanco e incluso shock hipovolémico si lo hace hacia el retroperitoneo. Los factores predisponentes para la ruptura son:

1. Ausencia de calcificación o calcificación incompleta.

2. Diámetro mayor de 2 centímetros.

3. HTA asociada.

4. Embarazo ${ }^{5-8}$.

Los aneurismas calcificados menores de $2 \mathrm{~cm}$ de diámetro y asintomáticos en pacientes normotensos no deben ser intervenidos, pero sí seguidos periódicamente para detectar cambios en el tamaño. El tratamiento quirúrgico está indicado en las siguientes situaciones ${ }^{9-11}$ :

- Aneurismas que producen isquemia renal.

- HTA secundaria al aneurisma.

- Aneurismas disecantes.

- Sintomatología local (dolor intenso, hematuria...).

- Mujeres en edad fértil y dispuestas a concebir.

- Aumento progresivo de tamaño en los controles periódicos.

- Aneurisma con trombo detectable en angiografía y evidencia de embolización.

En cuanto a las técnicas quirúrgicas ${ }^{10,12-14}$, puede realizarse una reparación in-vivo (resección con anastomosis termino-terminal ó colocación de prótesis) o ex-vivo (cirugía de banco y autotrasplante renal).

Lo expuesto anteriormente es extrapolable a los pacientes monorrenos con aneurisma de la arteria renal de su único riñón, si bien la expe-

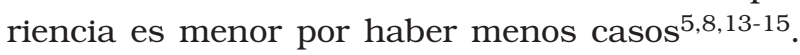
En ellos es fundamental un estudio exhaustivo pues los riesgos de cualquier actuación, tanto conservadora como intervencionista, son mayores ya que la pérdida de su riñón los aboca a una insuficiencia renal subsidiaria de tratamiento sustitutivo (diálisis/trasplante). 


\section{REFERENCIAS}

1. Seppala FE, Levey J. Renal artery aneurysm: case report of a ruptured clacified renal artery aneurysm. Ann Surg 1982 jan;48(1):42-44.

2. Tham G, Ekelund L, Herrlin K, Lindstedt EL, Olin T, Bergentz SE. Renal artery aneurysms. Natural history and prognosis. Ann Surg 1983 mar;197(3):348-352.

3. Bonamigo TP, Erling N Jr, Faccini FP. Rupture of a sacular renal artery aneurysm: report of a case. Surg Today 2002;32(8):753-755.

4. Njinou Ngninkeu B, Eucher P, Vandenbossche P, Lacrosse M, Van Cangh PJ, Lorge F. Ruptured aneurysm of the renal artery: a rara cause of macroscopic hematuria. Prog Urol 2002 jun; 12(3):454-458.

5. Dayton B, Hegerson Rb, Sollinger Hw, Acher Cw. Ruptured renal artery aneurysm in an pregnant uninephric patient: successful ex-vivo repair and autotransplantation. Surgery 1990 jun; 107(6):708-711.

6. Lacroix H, Bernaerts P, Nevelsteen A, Hanssens M. Ruptured renal artery aneurysm during pregnancy: successsful ex-situ repair and autotransplantation. J Vasc Surg 2001 jan;33(1):188-190.

7. Meabed AH, Onuora VC, Al Turki M, Koko AH, Al Jawini N. Rupture of a renal artery aneurysm in pregnancy. Urol Int 2002;69(1):72-74.

8. Smith JA, Maclish DG. Postpartum rupture of a renal artery aneurysm to a solitary kidney. Aust N Z J Surg 1985 jun;55(3):299-300.
9. Mc Carron JP Jr, Marshall VF, Whitsell JC. 2nd ; Indications for surgery on renal artery aneurysms. J Urol 1975 aug;1114(2):177-180.

10. Dzsinich C, Gloviczki P, Mckusik MA et al. Surgical management of renal artery aneurysm. Cardiovasc Surg 1993 jun; 1(3):243-247.

11. Lumsden AB, Salam TA, Walton KG. Renal artery aneurysm: a report of 28 cases. Cardiovasc Surg 1996 apr:4(2): 185-189.

12. Toshino A, Oka A, Kitajima $\mathrm{K}$ et al. Ex vivo surgery for renal artery aneurysms. Int J Urol 1996 nov;3(6):421-425.

13. Hidai H, Ide K, Fujishima S. In situ and ex situ renal artery aneurysmectomy in the solitary kidneys. J Urol 1980 aug; 124(2):274-278.

14. Sridhar KN, Wickham JE, Vijayan P. Ruptured renal artery aneurysm in a solitary kidney. Eur Urol 1983;9(4):252253.

15. Harada T, Nishizawa O, Tsuchida S. Renal artery aneurysms in solitary kidney of woman with potentiality of pregnancy. Urology 1984 jun;23(6):578-581.

Dr. J. Rodríguez Corchero

C/ Cueva del Gato, 3 - blq. 6 - $3^{\circ} \mathrm{A}$

41020 Sevilla

(Trabajo recibido el 16 diciembre de 2003) 